\title{
SIDERITIS ARBORESCENS SALZM. EX BENTHAM Y SIDERITIS $X$ GADITANA ROUY EN ANDALUCIA OCCIDENTAL
}

\author{
O. SOCORRO, M.L. ZAFRA \& I. TARREGA
}

RESUMEN: Se describen los caracteres taxonómicos de las distintas subespecies de Sideritis arborescens Salzm. ex Bentham y de Sideritis x gaditana Rouy. Se incluye además para cada taxon, tipo, distribución y material estudiado en Andalucia occidental.

SUMMARY: It is described at the report the taxonomicous characters of the different subespecies of Sideritis arborescens Salzm. ex Bentham and of Sideritis $x$ gaditana Rouy. It is also included for each taxon, type, distribution and material studied from West Andalucía.

Dentro del género Sideritis L., la sección Sideritis (= secc. Eusideritis Bentham Lab. Gen. Sp. 577. 1834 ) con distribución mediterráneo occidental y fundamentalmente representada en la Península Ibérica, se caracteriza por agrupar a plantas perennes sufruticosas, cálices no bilabiados con dientes subiguales o iguales, brácteas sésiles, con base ancha, amplectantes, dentadas y distintas de las hojas, y por sus pólenes tetracolpados.

Dicha sección está representada en Andalucía Occidental por 5 especies: S. incana L., S. grandiflora Salzm. ex Bentham, S. hirsuta L., S. arborescens Salzm. ex Bentham y S. x gaditana Rouy. De ellas estudiamos S. arborescens y S. X gaditana.

Sideritis arborescens Salzm. ex Bentham, Lab. Gen. Sp., 579 (1834)

Esta especie es indicada por Heywood (1972: 140) para el $S$ de España, pero lo realmente cierto es que este taxon se distribuye por el C, SE y SW de la España peninsular, $S$ de Portugal y $N$ de Africa. Este taxon, como la mayoría de los incluidos en su género, muestra un cierto polimorfismo, aunque pueden diferenciarse distintas poblaciones dentro de él.

La gran variabilidad que hemos observado en el material estudiado y que afectan sobre todo a tipo de indumento, tomento, glándulas, a forma, longitud y anchura de las hojas, a tomento y longitud de las brácteas, tomento y longitud de los cálices y color de la corola, motiva que estemos de acuerdo con Font Quer (1924: 34), Heywood (1.c.) y Borja (com. pers.) en estimar a las distintas poblaciones como integrantes de una sola especie y no darles el rango específico a cada una de ellas.

Font Quer (1.c.) atribuyó a estas poblaciones categoría de variedad, pero compartimos el criterio de Heywood (1.c.) pudiendo distinguirse cuatro subespecies: subsp. arborescens, subsp. perezlarae Bor- 
ja, subsp. paulii (Pau) P.W. Ball ex Heywood y subsp. luteola (Font Quer) P.W. Ball ex Heywood.

La subsp. arborescens se caracteriza por su revestimiento piloso doble, glándulas sentadas y pedunculadas, verticilastros 6-10 flores distantes o aproximados, cáliz que no sobrepasa los $8 \mathrm{~mm}$ en flor y los $9 \mathrm{~mm}$ en fruto, corola amarillo-pálida depasando el cáliz y núculas anchamente oblongas de $2,4-2,5 \times 1,8-2 \mathrm{~mm}$. Dentro de ella hemos observado ejemplares con ramas entre verticilastros y cálices hirsutos, los cuales corresponden a lo que, según Font Quer (1.c.: 33), Pau describió como S. hirsutoides. En la bibliografía conocida de Pau no hemos localizado tal especie por lo cual la consideramos por el momento como nomen nudum. Estimamos que al no existir en los mismos variaciones en cuanto a caracteres esenciales, por presentarse de forma esporádica y conviviendo con el tipo, no merecen destacarse con categoría taxonómica alguna. Se distribuye por la zona litoral de las provincias de Cádiz, Málaga y Granada.

La subsp. perezlarae, aunque su área se solapa en parte con la subespecie anterior, consideramos acertado el nivel taxonómico dado por Borja (1983: 278), y se separa de ella por su revestimiento piloso uniforme, glándulas sentadas, hojas y brácteas glabras. Se localiza en la zona litoral de la provincia de Cádiz.

La subsp. paulii, se separa fundamentalmente de la subsp. arborescens por la longitud de sus cálices y por sus núculas estrechamente oblongas $2,5 \times 1,5 \mathrm{~mm}$. Se distribuye por zonas interiores del $\mathrm{C}$ (Toledo y Ciudad Real) y S (Jaén, Córdoba, Sevilla y Huelva) de la Península Ibérica.

Por último, la subsp. luteola se distingue de las anteriores, por sus hojas lineares, estrechas, enteras o escasamente crenado-dentadas, verticilastros de menor diámetro y corolas rosadas. Aunque indicada por Heywood (1.c.) para la provincia de Granada, no se localiza en la misma, tratándose de un endemismo almeriense.

A continuación incluimos una clave para la separación de las tres subespecies con representación en Andalucía Occidental, y además los caracteres diagnósticos y distribución de cada una.

1. Con sólo glándulas sentadas; hojas glabras y glandulosas; brácteas

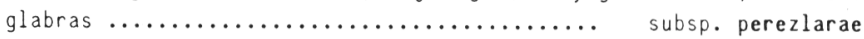

1. Con glándulas sentadas y pedunculadas; hojas subglabras a vellosas y glandulosas; brácteas subglabras a pubescentes y glandulosas....

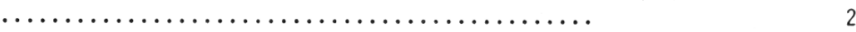

2. Cáliz 7 - $8 \mathrm{~mm}$ en flor, hasta $9 \mathrm{~mm}$ en fruto; núculas $2,4-2,5 \times$

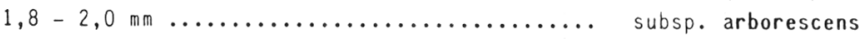

2. Cáliz 8 - $10 \mathrm{~mm}$ en flor, hasta $11 \mathrm{~mm}$ en fruto; núculas $2,5 \times 1,5$

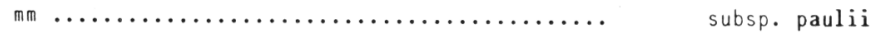

subsp. arborescens

S. hirsutoides Pau nomen nudum

S. arborescens var. typica Font Quer Trab. Mus. Ci. Nat. Barcelona 5(4): 34 (1924).

Entrenudos de las ramas con pelos cortos, incurvados, con dos caras glandulosas pubérulas y las otras dos subglabras; los de la inflorescencia, subghlabros a subhirsutos, con pelos más largos, incurvados y semipatentes. Hojas subglabras a vellosas y glandulosas. Ver- 
ticilastros 6 - 10 flores, distantes o aproximados. Brácteas subglabras a pubescentes y glandulosas, las superiores igualando o poco más cortas que los cálices y dientes con espina corta a lo sumo de $1 \mathrm{~mm}$. Cálices $7-8 \mathrm{~mm}$ en flor, hasta $9 \mathrm{~mm}$ en la fructificación, dientes con espina corta 0,5 mm. Núculas de 2,4 - 2,5 x 1,8 - 2,0 mm. $(\mathrm{K}), \mathrm{n} \cdot \mathrm{v}$.

Tipo: In hispaniae monte Gibraltarico, Broussenet \& Salzmann

Distribución: $\mathrm{S}$ de la Península Ibérica y $\mathrm{N}$ de Africa.

Material estudiado: CADIZ: La Línea de la Concepción, V.1965, Borja (MA 185912); Algeciras, IV.1970, J. Hurtado (SEV 99636). GIBRALTAR: In rupibus gibraltariae, 29.III.1841, Lemann (BM); idem, 23.IV.1841, Leman (BM) .

subsp. perezlarae Borja, Anales Jard. Bot. Madrid 40(1): 278 (1983)

Entrenudos de las ramas y de la inflorescencia con pelos cortos, recios, incurvados, con dos caras pubérulas o subglabras y las otras dos glabras y glandulosas. Hojas glabras y glandulosas. Verticilastros 6 flores, distantes. Brácteas glabras, las superiores más cortas que los cálices y dientes con espina corta a lo sumo de $1 \mathrm{~mm}$. Cálices 6 - $7 \mathrm{~mm}$ en flor, hasta $8 \mathrm{~mm}$ en la fructificación, dientes con espina corta $0,5 \mathrm{~mm}$. Núculas $2,5 \times(1,5-) 1,8 \mathrm{~mm}$.

Tipo: Barbate (Cádiz), arenas subcosteras en pinares de Pinus pinea, 10.VI.1981, Borja (MA 240430).

Distribución: SW de la Península Ibérica (prov. de Cádiz).

Material estudiado: CADIZ: Tarifa, Punta Palomas, 18.IV.1969, D.M.C. Brinton-Lee (SEV 83091); San Roque, Pinar del Rey, 30.IV.1980, B. Molesworth Allen (SEV 71861); Caños de Meca, 21.V.1980, F. Amor \& A. Barroso (GDA).

subsp. paulii (Pau) P.W. Ball ex Heywood, Bot. Jour. Linn. Soc. 65: 355 (1972)

S. paulii Pau, Bol. Soc. Esp. Hist. Nat. 21: 151 (1921).

S. arborescens var. paulii (Pau) Font Quer, Trab. Mus. Ci. Nat. Barcelona, 5(4): 34 (1924).

Entrenudos de las ramas con pelos cortos, incurvados, con dos caras glandulosas pubérulas y las otras dos subglabras, los de la inflorescencia con pelos más largos, incurvados y semipatentes. Hojas subglabras a vellosas y glandulosas. Verticilastros 6 - 10 flores, distantes. Brácteas subglabras a pubescentes y glandulosas, las superiores ocultando los cálices y dientes con espina de $(0,5-) 1,0-2,0 \mathrm{~mm}$. Cálices 9 - $10 \mathrm{~mm}$ en flor, hasta $11 \mathrm{~mm}$ en la fructificación, dientes con espina larga $1,0-2,0 \mathrm{~mm}$. Núculas $2,5 \times 1,5 \mathrm{~mm}$.

Tipo: Córdoba, 1900, Paul \& Arozarena (MA 100273 lectótipo).

En el herbario del Real Jardín Botánico de Madrid, hemos hallado un pliego (MA 100273) que contiene dos fragmentos muy incompletos. En uno se observa dos verticilastros y en el otro una pequeña porción de los entrenudos y dos verticilastros. Lleva además, un recorte del Bol. Soc. Esp. Hist. Nat. en el que se aprecia su descripción y una 
etiqueta manuscrita en la que puede leerse: "Según muestra de H. cordobés, esta forma crece en Siles de Segura. Falta el otro fragmento, porque me lo pidió Quer."

Font Quer (1.c.: 35) indica acerca de este taxón: "...les dues mostres de l'Herbari Pau són solamente de les sumitats florides, amb els verticillastres i una parella de fulles superiors". Ello coincide con lo observado por nosotros en dicho pliego, de ahí que lo elijamos, a pesar de no ser lo necesariamente completo, como tipo pues en él pueden apreciarse buena parte de sus caracteres esenciales.

Distribución: C y SW de la Península Ibérica.

Material estudiado: CORDOBA: Sierra de Villanueva de Córdoba, 10.VII. 1963, Rivas Goday \& Demetrio (MAF 72656). HUELVA: Sierra de Aracena, entre Valdezufre y Aracena, 18.VI.1978, J. Rivera (SEV 47830); ídem, 5.VII.1983, Socorro \& Garcia-Granados (GDA); Sierra de Aracena, entre Corteconcepción y Puerto Moral, 6.VI.1979, J. Rivera \& B. Cabezudo (SEV 47832); ídem, 6. VII.1983, Socorro \& Garcia-Granados (SEV 99637); Sierra de Aracena, Puerto Moral, La Umbría, 6.VII.1979, J. Rivera (SEV 47831 y 57796); Sierra de Aracena, entre Puerto Moral y Corteconcepción, 7.VII.1978, J. Rivera (SEV 47833 y 57797). SEVILlA: Coripe, río Guadalete, 15.IV.1977, E. Ruiz de Clavijo (SEV 28928).

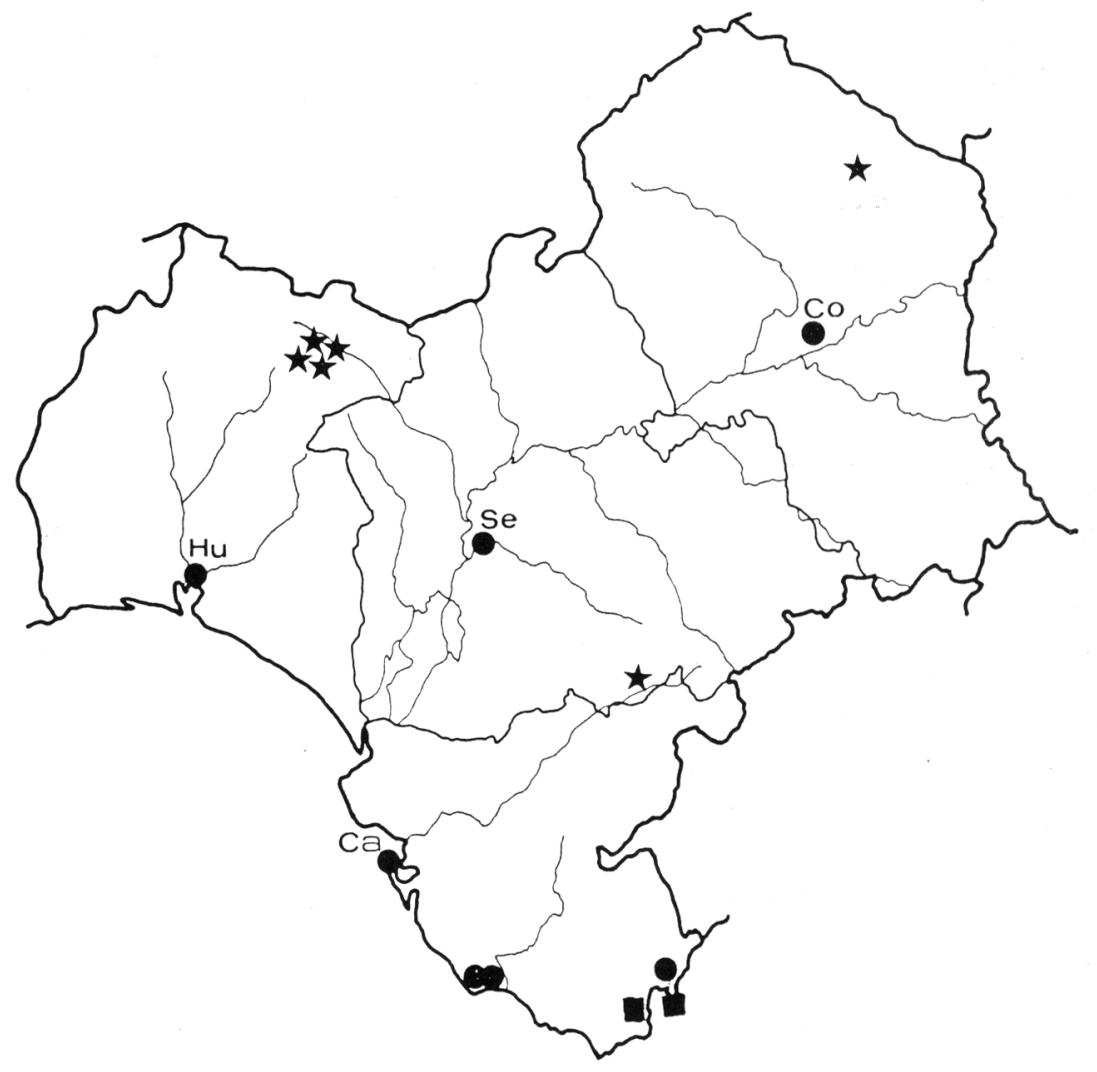

\footnotetext{
Mapa 1.- Localidades estudiadas de S. arborescens Salzm. ex Bentham

subsp. arborescens; subsp. perezlarae Borja; $\boldsymbol{\gamma}$ subsp. paulii (Pau)

P.W. Ball ex Heywood.
} 
Sideritis x gaditana Rouy, Ill. Pl. Eur. Rar., 17:137, t.417 (1902)

S. hirsuta L. var. laniculata Pérez Lara, Mem. Real Soc. Esp. Hist. Nat. 2: 46 (1903).

S. arborescens Salmz. ex Bentham x S. hirsuta L.

Planta sufruticosa, hasta $50 \mathrm{~cm}$. Verde a verde-grisácea, ramificada, ramas ascendentes y erectas o flexuosas, glandulosas, en la base subglabras a crispado-vellosas, entre verticilastros laxamente hirsutas. Hojas lineares, linear-lanceoladas u oblongo-lanceoladas, glandulosas, subglabras a crispado-vellosas, atenuadas y con bordes enteros y ciliados en su base, en su mitad superior crenado-dentadas. Verticilastros 6 - 10, distantes, 6 - 10 flores. Brácteas glandulosas - subglandulosas, subglabras a laxamente hirsutas, inciso-dentadas, dientes no espinosos, las inferiores ovales o acorazonado-ovales 6 $-16(-18) \times 11-13(-15) \mathrm{mm}$, las restantes acorazonado-ovales o acorazonado-semiorbiculares 5 - $10(-11) \times 11$ - 15(-16,5) mm. Cálices 7 $10 \mathrm{~mm}$, campanulados, hirsutos, glandulosos, dientes lanceolados, subespinosos y desiguales. Corola (9-)10 - $11 \mathrm{~mm}$, blanca o labio superior blanco e inferior amarillento.

Tipo: Cádiz. Puerto de Santa María, coteaux vers Jerez, 6.IV. 1887, G. Rouy (LY, lectótipo), v. foto.

En el herbario de la Universidad de Lyon (LY) se conserva un pliego del propio Rouy que contiene tres ejemplares. Se elige como lectótipo el ejemplar situado en el extremo derecho.

Rouy (1902: 137) indica que su S. gaditana es intermedia entre S. arborescens y S. scordioides L. Aunque no hemos podido estudiarla a partir del tipo, sólo hemos contado con una fotografía del mismo, si lo hemos hecho a partir de ejemplares recolectados en su localidad clásica, observando que se trata de un híbrido en que uno de los padres corresponde a S. arborescens, mientras que el otro a S. hirsuta.

Pérez Lara (1903: 46) describe de diversas localidades de la provincia de Cádiz la var. laniculata de S. hirsuta. Hemos comprobado al estudiar el material de dicho autor existente en MAF, que dicha variedad corresponde a S. x gaditana.

Como consecuencia de nuestras propias herborizaciones y del análisis del material de los herbarios SEV, MA, MAF, hemos observado una cierta variabilidad (dominio de un padre sobre el otro y viceversa), y ello debido a posibles retrocruzamientos. Tales variaciones afectan al tomento y a la longitud de brácteas, cálices y corola.

Distribución: SW de la Península Ibérica.

Material estudiado: CADIZ: Jerez, 7.IV.1878. Pérez Lara (MAF 32422); Puerto Real, 19.IV.1876, Pérez Lara (MAF 32420); entre Jerez de la Frontera y Puerto de Santa María, 9.III.1978, J. Pastor, S. Talavera \& B. Valdés (SEV 99644); entre Arcos de la Frontera y Bornos, Puente sobre el Guadalete, 16.IV.1980, A Martínez (SEV 99643); Jerez, 11.V.1978, Talavera, Devesa \& Pastor (SEV 99642)); Bornos, 31.V.1969, E.F. Galiano, Silvestre \& Valdés (SEV 99641); Jerez, Sierra de San Cristobal, 5.VII.1983, Socorro \& GarcíaGranados (SEV 99640); a 2 Km de Bornos hacia Arcos, 4.VII.1983, Socorro \& García-Granados (SEV 99639); Arcos, Cuesta de la Escalera, 5.VII.1983, Socorro \& García-Granados (SEV 99638); Grazalema, VI.1961, Borja (MAF 102453 y 68434). SEVIlla: Carmona, 30.IV.1881, G. Fragoso (MAF 32423). Alrededores de Mairena del Alcor, 26.IV.1975, R. de Clavijo (SEV 99645); Alcalá de Guadaira, 8.IV.1973, B. Cabezudo (SEV 99646); entre El Gandul y Trujillo, 2.V.1975, B. Cabezudo, S. Talavera \& B. Valdés (SEV 99647); Carmona, Ermita Sta. Ma de Gracia, 24.IV.1979, P. Candau, I. Fernández \& T. Luque (SEV 99648); Villaverde del Río, 21.V.1982, Morales, Medina \& Molina (SEV 99651). HUElva: Hinojos, 29.v.1976, B. Cabezudo, Domínguez, Gallego, Ramos \& Talavera (SEV 99649). 


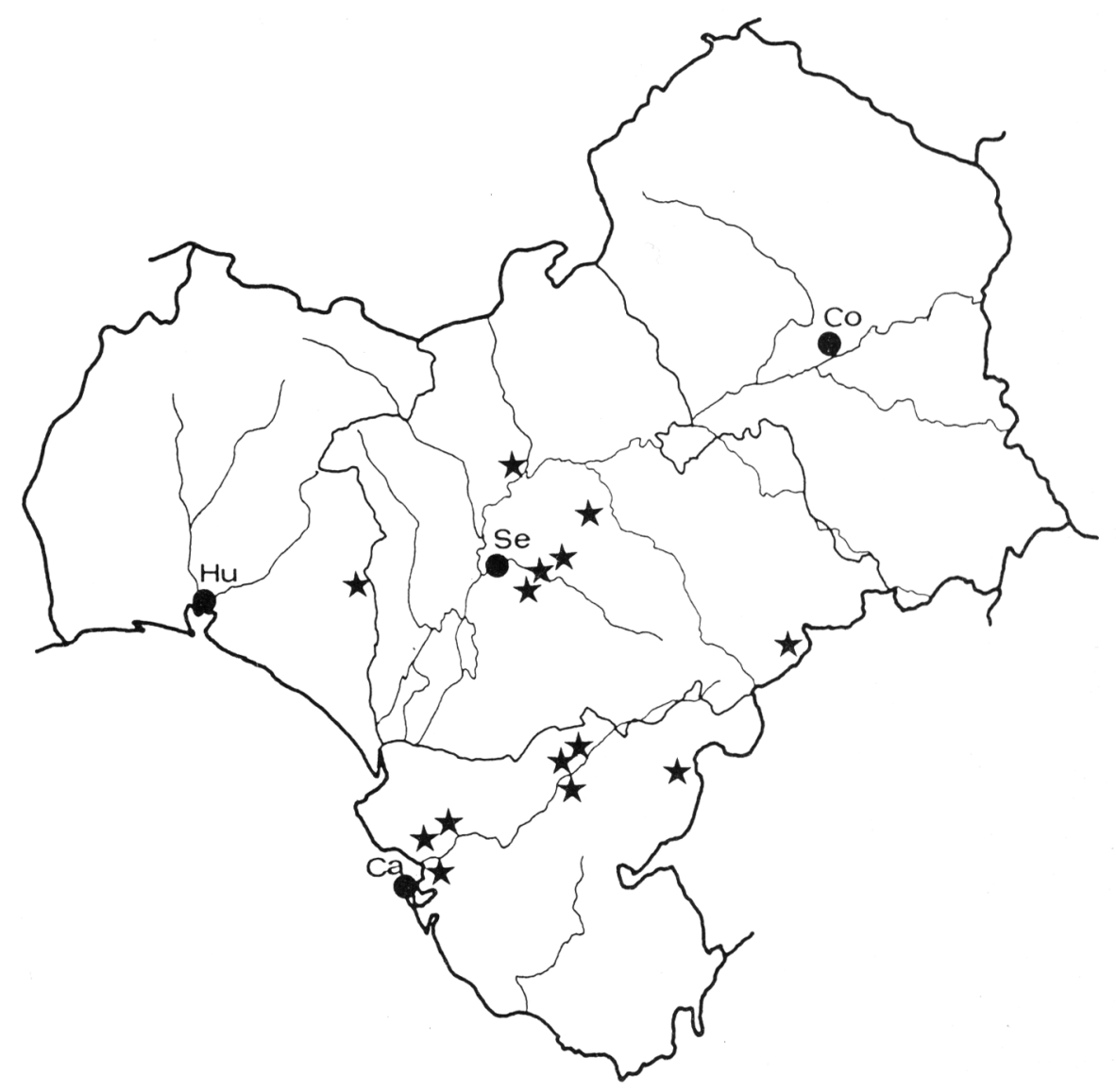

Mapa 2.- Localidades estudiadas de S. x gaditana Rouy.

BIBLIOGRAFIA

BORJA, J. -1983- Sideritis arborescens Salzm. ex Bentham subsp. perezlarae Borja subsp. nov. Anales Jard. Bot. Madrid, 40(1): 278.

FONT QUER, P. -1924- Estudis sobre morfologia i nomenclatura de les sideritis. Trab. Mus. Ci. Nat. Barcelona, 5(4): 3-35.

HEYWOOD, V.H. -1972- Sideritis, in T.G. Tutin \& al. (eds.) Flora Europaea, 3: 138-143. Cambridge.

PEREZ LARA, J.M. -1903- Florula Gaditana. Addenda et Emendanda. Mem. Real Soc. Esp. Hist. Nat., 2: 5-62.

ROUY, G.C.C. -1902- Illustrationes plantarum Europae rariorum. 17. Paris. 